\title{
Evolution of Reciprocity in Asymmetric International
}

\author{
Environmental Negotiations*
}

\author{
Marius I. Ochea ${ }^{\dagger} \quad$ Aart de Zeeuw ${ }^{\ddagger}$
}

24th September 2013

\begin{abstract}
We study the evolutionary success of a generalised trigger strategy within an asymmetric, $n$-player Prisoner's Dilemma environment, with application to the evolution of cooperation in international environmental negotiations. Our results suggest that there exist regions in the relevant parameter space - i.e. costs and benefits, low and high titfor-tat thresholds, probability of continued interaction - such that (partial) cooperation may emerge as long-run attractor of the evolutionary dynamics in these asymmetric social dilemmas.
\end{abstract}

JEL classification: C72, C73, D62, H77

Keywords: International Environmental Agreements, tit-for-tat, asymmetric Prisoner's Dilemma, replicator dynamics

*Part of Marius Ochea's work on the paper was financed by the project "SENSE", funded by the TECT program of the European Science Foundation, and part of Aart de Zeeuw's work on the paper was financed by the project "Improving international cooperation on emission abatement", funded by the NORKLIMA program of the Norwegian Research Council.

${ }^{\dagger}$ Amsterdam School of Economics and CeNDEF, University of Amsterdam, Valckenierstraat 65-67, 1018 XE Amsterdam, The Netherlands. M.I.Ochea@uva.nl

‡Tilburg Sustainability Center, Department of Economics, Tilburg University, P.O. Box 90153, 5000 LE Tilburg, the Netherlands, Beijer and CESifo. A.J.deZeeuw@uvt.nl 


\section{Introduction}

The history of the Kyoto Protocol has shown how difficult it is to maintain and implement International Environmental Agreements, with the purpose to internalize transboundary externalities such as climate change. A tragedy of the commons occurs whenever there exist a wedge between individually and collectively rational actions. The literature has mainly focused on the stability properties of a coalition in a two-stage non-cooperative game and has shown that free-rider incentives dominate incentives to cooperate, so that stable coalitions are small (Barrett (1994), Finus (2003)). When countries differ in their costs and benefits of emission reductions (or abatement), the stable coalition can be larger but total abatement remains low as compared to the full-cooperative outcome (McGinty (2007), Fuentes-Albero and Rubio (2010), Pavlova and de Zeeuw (2013)).

The history of the Kyoto Protocol has also shown that countries stop believing that a general agreement on emission reductions can be achieved and start searching for alternatives. For example, some countries that did not ratify the Kyoto Protocol at some stage suggested to focus on technology development instead. If the technological progress becomes generally available, technology agreements may indeed improve the situation but the full-cooperative outcome is not guaranteed (e.g. Hoel and de Zeeuw (2013)). Leadership may be another option. The idea is that some countries start with emission reductions and other countries follow. One may say that the Kyoto protocol was saved for some time by the European Union trying to take up a leadership role. Furthermore, at the Conference of the Parties (COP 15) in Copenhagen in 2009 a few other countries took the lead with the idea to build emission reductions bottom up, instead of top down via an unanimously agreed upon plan. To take this one step further, reciprocity may be the answer. The folk theorem in game theory says that players can keep each other on a cooperative path by starting with cooperation and using trigger strategies that sufficiently punish deviations. In a world with different strategies, the question is whether this type of strategies can survive and induce cooperation. 
Various mechanisms have been proposed in the literature to deal with social dilemmas focusing on direct or indirect reciprocity through rewards or punishments and on spatial or local interaction (Nowak and Sigmund (2006)). In particular, conditional trigger strategies in repeated Prisoner's Dilemma games have been shown to be effective in overcoming the typical gloomy picture of perpetual deviations. Models on the evolution of simple behavioral rules in pairwise interactions with symmetric payoff structures show that cooperative behavior can be generated. However, this theoretical framework has its limitations as social dilemmas are rarely two-person games and asymmetries in payoffs are ubiquitous. The international game of emission reductions usually is a Prisoners's Dilemma game but it has many players and the payoffs to the countries are clearly asymmetric. The purpose of this paper is to analyze the evolution of "tit-for-tat" strategies in a world that also contains strategies that always defect, and to show in which situation cooperation will be generated.

Our paper is related to a paper by Boyd and Richerson (1988). Their basic model is similar to a typical model in the literature on International Environmental Agreements. It has $n$ players but it assumes symmetry. In our model the countries that interact are randomly drawn from a population with two types of countries that differ in their costs and benefits of emission reductions. Following Boyd and Richerson (1988) we have two possible strategies: always defect and a "tit-for-tat" strategy that starts cooperating and continues cooperating if sufficiently many countries in the interacting group cooperate. Since we have asymmetry, in our model the trigger strategies of the two types may differ. The dynamical evolution is described by replicator dynamics: if a strategy is successful in the interacting group, the share of this strategy in the population increases. We will show that the parameter space has areas where full-cooperation will result and areas where no cooperation will result. We will show that sufficiently high initial fractions of "tit-for-tat" strategies, sufficiently high thresholds in the trigger strategies and a sufficiently high probability of continued interaction induces full cooperation at the end of the process.

Other papers have studied the symmetric $n$-player repeated Prisoner's Dilemma game as 
well. Diekmann and Manhart (1989) show with simulations that the "tit-for-tat" strategy could be relatively successful, even in large groups. Molander (1992) finds that a generalized reciprocity rule may co-exist with defectors in the limit set of the evolutionary dynamics. Lindgren and Johanson (2002) run a simulation study of Boyd and Richerson (1988) and Molander (1992), using finite automata, and suggest that strategies with memory two or higher (i.e. conditioning on two or more past moves of the opponents) could escape the defection fixed points. Rieskamp and Todd (2006) study the role of asymmetric payoffs in a two-player repeated Prisoner's Dilemma game and show, via simulations, that the three bestperforming strategies differ from the best-performing strategy in a symmetric set-up. On the methodological side, our contribution is the analysis of an asymmetric $n$-player repeated Prisoner's Dilemma game.

Other papers have studied International Environmental Agreements from an evolutionary game perspective as well. Breton et al. (2010) analyze a game with stock externalities and assume that each signatory has to punish non-signatories for their irresponsible behavior. Countries become asymmetric because the costs of punishing and the costs of being punished differ. Replicator dynamics favors the successful strategy and leads to an equilibrium share of signatories that depends on the parameters of the problem. McGinty (2010) analyses a standard game of International Environmental Agreements but assumes that the marginal benefit of total abatement differs between signatories and non-signatories, with possibly different types as well. The evolutionary equilibrium determines the size of the coalition and depends on whether the basic game becomes a Prisoner's Dilemma, a Coordination Game or a Hawk-Dove Game. Our contribution is the study of the evolution of reciprocity, in the form of "tit-for-tat" strategies, in the international game of emission reductions as an asymmetric n-player Prisoner's Dilemma.

Section 2 introduces the model with the asymmetric trigger strategies. The repeated game payoffs are computed in the Appendix, whereas Section 3 reports numerical results of the continuous-time replicator dynamics for different parameter values. Concluding remarks 
and future directions of research are presented in Section 4.

\section{The model}

International Environmental Agreements are usually modeled as a two-stage game where in the first stage countries decide whether they want to sign the agreement or not and where in the second stage the emission reductions are decided in a game between the coalition of signatories and the individual outsiders (Barrett (1994), Finus (2003)). The (symmetric) $n$ countries have the discrete choice to abate or not and have $\operatorname{costs} c$ of their own abatement and benefits of total abatement. If all the countries abate, the (maximal) benefits are $B$ for each country. Individually each country generates benefits $B / n$ and if $c>B / n$, it is not individually rational to abate. However, if $B>c$, it is collectively rational to abate. Moreover, if $i B / n>c$, it is rational for the group of $i$ countries to abate. The smallest $i$ for which this holds is the size of the stable coalition: if the coalition gets smaller, it has no reason to form, and adding another country to the coalition decreases the net benefits of the group. This implies that only the fraction $(i / n)$ of maximal benefits $B$, that just covers the cost $c$, is realized with the stable coalition and this is usually not a very good result. Therefore we investigate whether it is possible to achieve maximal benefits or full cooperation when countries change their strategies and do not try to reach an agreement. Instead we investigate the option that part of the population of countries adopts a "tit-for-tat" strategy starting with cooperation or abatement and continuing with cooperation if sufficiently many other countries cooperate. The other countries in the population do not abate or defect. However, if the "tit-for-tat" strategy is successful, other countries adopt this strategy as well and in this process "tit-for-tat" strategies may be attractors and at the end full cooperation may be achieved. In this analysis it is assumed that in each period of time only part of the population interacts and plays a repeated game with some probability of continuation. After the repeated game ends, the distribution of the strategies in the population is adjusted and 
the process continues with a new random draw of a part of the population. We will build the model in steps.

\subsection{Prisoner's Dilemma}

The basic game with two countries is a standard Prisoner's Dilemma (where $C$ denotes cooperation or abatement and $D$ denotes defection or no abatement) that can be represented by a bi-matrix where the first entries are the net benefits of country 1 and the second entries are the net benefits of country 2 :

$$
\left[\begin{array}{ccc} 
& C & D \\
C & B-c, B-c & B / 2-c, B / 2 \\
D & B / 2, B / 2-c & 0,0
\end{array}\right] .
$$

The assumptions in the text above imply that $B / 2>B-c>0>B / 2-c$ and $2(B-c)>B-c$ so that this game is a standard Prisoner's Dilemma. Defection $D$ is the dominant action, $(D, D)$ is the Nash equilibrium and $(C, C)$ is collectively rational.

An n-player Prisoner's Dilemma (e.g. Taylor (1976), Schelling (1978)) is specified as follows. Let $V(C \mid i)$ and $V(D \mid i)$ denote the individual payoffs if $i$ of the $n$ players cooperate. These payoffs should have the following properties:

$$
V(D \mid i)>V(C \mid i+1), i=0,1, \ldots, n-1,
$$

which means that defection $D$ is the dominant action,

$$
\begin{aligned}
& V(D \quad \mid \quad i+1)>V(D \mid i), i=0,1, \ldots, n-1, \\
& V(C \quad \mid \quad i+1)>V(C \mid i), i=0,1, \ldots, n-1,
\end{aligned}
$$

which means that if a player switches from defection to cooperation, every other player is 
better off, and

$$
(i+1) V(C \mid i+1)+(n-i-1) V(D \mid i+1)>i V(C \mid i)+(n-i) V(D \mid i),
$$

which means that the average payoff (or fitness) increases if one player switches from defection to cooperation.

If $n=2$ these properties generate a slightly stronger form of the Prisoner's Dilemma than usual for two players because they also require that the off-diagonal average payoff is higher than the payoff in the Nash equilibrium. In any case, for the $n$-country game of emission reductions it holds that $V(C \mid i)=i B / n-c$ and $V(D \mid i)=i B / n$ and the assumptions in the text above imply that $i B / n>(i+1) B / n-c,(i+1) B / n>i B / n$ and $(i+1)((i+1) B / n-c)+(n-i-1)(i+1) B / n>i(i B / n-c)+(n-i) i B / n$ (because $B>c)$ so that the three properties hold. This model is precisely the basic model in Boyd and Richerson (1988).

Since the countries are asymmetric in their costs and benefits of emission reductions, we introduce an asymmetry into the basic model. We assume that there are two types in the population, denoted by $I$ and $J$. Countries of type $I$ contribute $B / n$ to the benefits of abatement and have $\operatorname{costs} c_{h}$. Countries of type $J$ contribute $b / n$ to the benefits of abatement and have $\operatorname{costs} c_{l}$, where $B>b$ and $c_{h}>c_{l}$. One may say that countries of type $I$ are developed (Annex I) countries that have high emissions but also high costs of emission reductions and that countries of type $J$ are less developed (Annex II) countries that have low emissions but also low costs of emission reductions. If $i$ and $j$ are the number of countries of type $I$ and $J$, respectively, that cooperate, the net benefits for country $i$ of type $I$ are

$$
\begin{aligned}
& V\left(C_{i} \quad|\quad i| j\right)=\frac{B i+b j}{n}-c_{h}, \\
& V\left(D_{i} \quad|\quad i| j\right)=\frac{B i+b j}{n},
\end{aligned}
$$


and the net benefits of country $j$ of type $J$ are

$$
\begin{aligned}
& V\left(C_{j} \quad|\quad i| j\right)=\frac{B i+b j}{n}-c_{l}, \\
& V\left(D_{j} \quad|\quad i| j\right)=\frac{B i+b j}{n} .
\end{aligned}
$$

The game is played as follows. In each period of time, $n / 2$ ( $n$ even) countries are drawn randomly from each population, according to the binomial distribution. These countries play a repeated $n$-player asymmetric Prisoner's Dilemma, with the game above as the stage game. The game continues with probability $0<w<1$. After the repeated game ends, the fractions of type $I$ and $J$ in the total population are adjusted with replicator dynamics which we will describe below. First we attend to the possible strategies in the repeated game.

\section{$2.2 \quad$ Asymmetric trigger strategies}

The countries can have one of two strategies: always defect (no abatement) or a trigger strategy. For the case of two countries, a trigger strategy conditions the action of a country on the action(s) of the other country. The "tit-for-tat" strategy prescribes that a country starts cooperating and cooperates if the other country has cooperated one step earlier and defects if the other country has defected one step earlier. For the case of $n$ (symmetric) countries, Taylor (1976) introduced a generalization of "tit-for-tat" prescribing that a country cooperates if $\alpha$ or more of the other countries have cooperated one step earlier and otherwise defects. Note that this is a set of strategies since $\alpha$ can take values $1, \ldots, n-1$. Because of the asymmetry between the countries, we generalize this set of strategies further and introduce asymmetric

"tit-for-tat" strategies for conditional cooperation as follows. If $i$ and $j$ are countries of type $I$ and $J$, respectively, we define the "tit-for-tat" strategies TFT by:

$$
T F T_{\alpha_{1}, \beta_{1}}^{i}, T F T_{\alpha_{2}, \beta_{2}}^{j},\left\{\alpha_{1}, \beta_{1}, \alpha_{2}, \beta_{2}\right\} \in\left[0, \frac{n}{2}-1\right]
$$


TFT $T_{\alpha_{1}, \beta_{1}}^{i}$ : "start with $C_{i}$ and choose $C_{i}$ if at least $\alpha_{1}$ type $I$ and $\beta_{1}$ type $J$ countries cooperated in the previous round, otherwise choose $D_{i} "$,

TFT $T_{\alpha_{2}, \beta_{2}}^{j}$ : "start with $C_{j}$ and choose $C_{j}$ if at least $\alpha_{2}$ type $I$ and $\beta_{2}$ type $J$ countries cooperated in the previous round, otherwise choose $D_{j} "$.

The thresholds $\left\{\alpha_{1}, \beta_{1}, \alpha_{2}, \beta_{2}\right\}$ reflect the stringency of the reciprocative rules. The ordering of the thresholds $\left\{\alpha_{1}, \beta_{1}, \alpha_{2}, \beta_{2}\right\}$ yields four cases: (i) $\left\{\alpha_{1} \leq \alpha_{2}, \beta_{1} \leq \beta_{2}\right\}$, (ii) $\left\{\alpha_{1} \geq \alpha_{2}, \beta_{1} \geq \beta_{2}\right\}$, (iii) $\left\{\alpha_{1} \leq \alpha_{2}, \beta_{1} \geq \beta_{2}\right\}$ and (iv) $\left\{\alpha_{1} \geq \alpha_{2}, \beta_{1} \leq \beta_{2}\right\}$. In the sequel we focus on case (i) because it seems reasonable to assume that the countries of type $I$ (Annex I) are willing to cooperate in a less cooperative environment than the countries of type $J$ (Annex II). The analysis of the other cases is similar but since the analysis is tedious we will not include the other cases in this paper.

If the random draw yields $k$ countries of type $I$ and $l$ countries of type $J$ that use the trigger strategy, Figure 1 shows the paths of actions of these countries for the different positions of $k$ and $l$ with respect to the thresholds $\left\{\alpha_{1}, \beta_{1}, \alpha_{2}, \beta_{2}\right\}$.

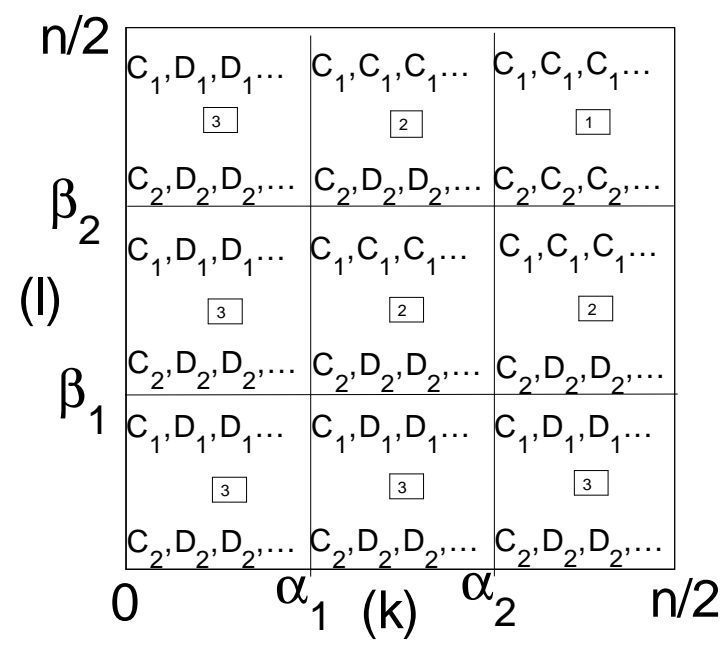

Figure 1: Paths of tit-for-tat play over all possible sampling configurations for the $\alpha_{1}<\alpha_{2}$, $\beta_{1}<\beta_{2}$ thresholds ordering

There are three possible outcomes. In the upper right corner (denoted by 1 ) there is full cooperation. In the square in the middle, plus the squares to the right and above the middle 
one (denoted by 2), countries of type $I$ always cooperate but countries of type $J$ switch to defection after the first step. In the other squares (denoted by 3) all countries switch to defection after the first step.

\subsection{Replicator Dynamics}

The countries in both parts of the population can have one of two strategies: always defect (no abatement), denoted by $A l l D^{i}, A l l D^{j}$, or the trigger strategy, denoted by $T F T_{\alpha_{1}, \beta_{1}}^{i}, T F T_{\alpha_{2}, \beta_{2}}^{j}$. We will refer to countries using the trigger strategy as reciprocators. The idea of replicator dynamics is that countries may switch to the other strategy if it proves to be better, which is reflected by the total net benefits $\Pi$ that the two strategies achieve in the interaction. It captures the idea that strategies that fare better than the average tend to spread in the population. Replicator dynamics models the evolution of the fractions of the trigger strategies $T F T_{\alpha_{1}, \beta_{1}}^{i}, T F T_{\alpha_{2}, \beta_{2}}^{j}$ in the two populations $I, J$. Let $\left(\rho_{1}, \rho_{2}\right)$ denote the shares of reciprocators in the two populations $I, J$, respectively. Formally, replicator dynamics is given by:

$$
\begin{aligned}
& \dot{\rho}_{1}=f_{1}\left(\rho_{1}, \rho_{2}\right)=\rho_{1}\left[\Pi_{T F T_{\alpha_{1}, \beta_{1}}^{i}}-\left[\rho_{1} \Pi_{T F T_{\alpha_{1}, \beta_{1}}^{i}}+\left(1-\rho_{1}\right) \Pi_{A l l D^{i}}\right],\right. \\
& \dot{\rho}_{2}=f_{2}\left(\rho_{1}, \rho_{2}\right)=\rho_{2}\left[\Pi_{T F T_{\alpha_{2}, \beta_{2}}^{j}}-\left[\rho_{2} \Pi_{T F T_{\alpha_{2}, \beta_{2}}^{j}}+\left(1-\rho_{2}\right) \Pi_{A l l D^{j}}\right],\right.
\end{aligned}
$$

or

$$
\begin{aligned}
& \dot{\rho}_{1}=\rho_{1}\left(1-\rho_{1}\right)\left(\Pi_{T F T_{\alpha_{1}, \beta_{1}}^{i}}-\Pi_{A l l D^{i}}\right)=\rho_{1}\left(1-\rho_{1}\right) \Delta \Pi_{i}, \\
& \dot{\rho}_{2}=\rho_{2}\left(1-\rho_{2}\right)\left(\Pi_{T F T_{\alpha_{2}, \beta_{2}}^{j}}-\Pi_{A l l D^{j}}\right)=\rho_{2}\left(1-\rho_{2}\right) \Delta \Pi_{j} .
\end{aligned}
$$

Note that the differences in net benefits between the reciprocative strategy and unconditional defection fully determines the dynamics of the replicator equations so that we only need to compute the payoff differentials $\Delta \Pi_{i}$ and $\Delta \Pi_{j}$ in order to characterize the attracting set of this system. 
The replicator dynamics (6), (7) has steady states on the boundary, that is $\left(\rho_{1}^{*}, \rho_{2}^{*}\right) \in$ $\{(0,0),(0,1),(1,0),(1,1)\}$, or in the interior, that is $0<\rho_{1}^{*}, \rho_{2}^{*}<1$, at zeros of the following system:

$$
\left[\begin{array}{c}
\Delta \Pi_{i}=0 \\
\Delta \Pi_{j}=0
\end{array}\right] .
$$

Summarizing, the game is played as follows:

(i) $n / 2$ countries are randomly drawn from each population $I, J$ and matched to play a repeated $n$-player asymmetric Prisoner's Dilemma

(ii) in each stage the countries can choose to cooperate (abate) $C$ or to defect (no abatement) $D$

(iii) the net benefits in each stage are given by (1)-(4)

(iv) the $n$-player asymmetric Prisoner's Dilemma continues with another stage with probability $w$

(v) the repeated game strategy set consists of two behavioral repeated game strategies $\left\{T_{F T}^{i} T_{\alpha_{i}, \beta_{i}}, T_{F T}^{j} T_{\alpha_{i}, \beta_{i}}^{j}, A l l D_{i}, A l l D_{j}\right\}:$ reciprocators and unconditional defectors

(vi) the probability of sampling a certain number of reciprocators or unconditional defectors from the populations $I, J$ follows the binomial distribution

(vii) after the repeated game ends, the countries get the opportunity to revise their strategy on the basis of the payoff differentials between the two behavioral rules

(viii) the fractions of each strategy in the populations $I, J$ are updated according to the replicator dynamics (6)-(7).

In Appendix A we compute the expected payoff differentials. The dynamics of the evolutionary system are fully described by the replicator dynamics (6), (7) and the payoff differentials (12), (13) of the trigger strategies $T F T_{\alpha_{1}, \beta_{1}}^{i}$ and $T F T_{\alpha_{2}, \beta_{2}}^{j}$ over the unconditional defection strategies. The system in $\rho_{1}$ and $\rho_{2}$ is highly non-linear and is very difficult to solve 
explicitly for a general parameterization. Therefore we will present a numerical analysis in the next section.

\section{Numerical results}

We will focus the analysis on the extreme cases where reciprocal countries either cooperate irrespective of the number of cooperators they are matched with or where they require all countries of one or both types to cooperate in the previous stage. We distinguish the following cases:

(a) $\alpha_{1}=\beta_{1}=\alpha_{2}=\beta_{2}=0$ : both types $I, J$ cooperate irrespective of the number of cooperators they are matched with,

(b) $\alpha_{1}=\beta_{1}=0, \alpha_{2}=\beta_{2}=\frac{n}{2}-1$ : type $I$ is very accommodative, whereas type $J$ is very restrictive,

(c) $\alpha_{1}=\alpha_{2}=\frac{n}{2}-1, \beta_{1}=\beta_{2}=0$ : both types $I, J$ care only about type $I$ 's cooperation,

(d) $\alpha_{1}=\beta_{1}=\alpha_{2}=\beta_{2}=\frac{n}{2}-1$ : both types $I, J$ are very restrictive (these are the TFT strategies used in Boyd and Richerson (1988) but now with asymmetries).

Notice that not all possible orderings can be investigated as the specific evolutionary dynamics are derived for the particular threshold ordering $\left\{\alpha_{1} \leq \alpha_{2}, \beta_{1} \leq \beta_{2}\right\}$. Given the complicated, non-linear structure of the dynamical system (6)-(7) we only report numerical results for these configurations of thresholds. The benchmark model parameterization is: $n=20, B=40, b=30, c_{h}=3, c_{l}=2$ and $w=0.8$. We will investigate the sensitivity for these benchmark parameter values later. 


\subsection{Cases (a), (b), (c) and (d)}

(a) $\alpha_{1}=\beta_{1}=\alpha_{2}=\beta_{2}=0$. Both type $I$ and type $J$ countries cooperate irrespective of the other countries' abatement decision. As can be seen in Figure 2a, most of the initial conditions are attracted to the $(0,1)$ equilibrium with type $J$ countries switching to the reciprocal strategy $T F T$ while type $I$ countries end up as unconditional defectors with strategy AllD. In this case, at the end of the evolutionary process, type $J$ countries keep each other on the cooperative path, whereas type $I$ countries just free-ride on the efforts of type $J$ countries.

(b) $\alpha_{1}=\beta_{1}=0, \alpha_{2}=\beta_{2}=\frac{n}{2}-1$. Type $I$ countries cooperate regardless of the structure of the group of countries they are matched with, while type $J$ countries only cooperate if all the other countries in the group cooperate. Depending on the initial conditions two possible equilibria emerge: $\left(0, \rho_{2}^{*}\right), \rho_{2}^{*} \in[0,1]$ and $(1,1)$, with a smaller basin of attraction for the latter equilibrium (Figure 2b). Full cooperation is thus possible provided that the two populations $I, J$ start with sufficiently high fractions of reciprocators. Compared with case (a), the very restrictive behaviour of type $J$ countries opens up the possibility of full cooperation.

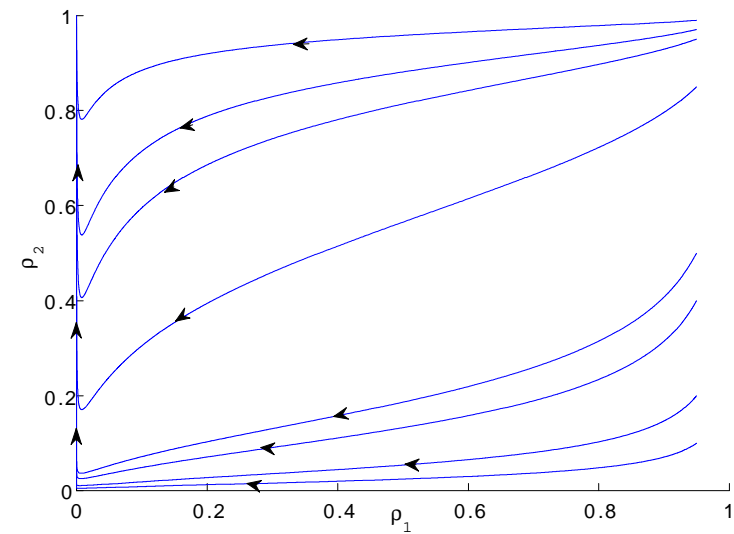

(a) Phase plot $\alpha_{1}=0, \alpha_{2}=0 ; \beta_{1}=0, \beta_{2}=0$

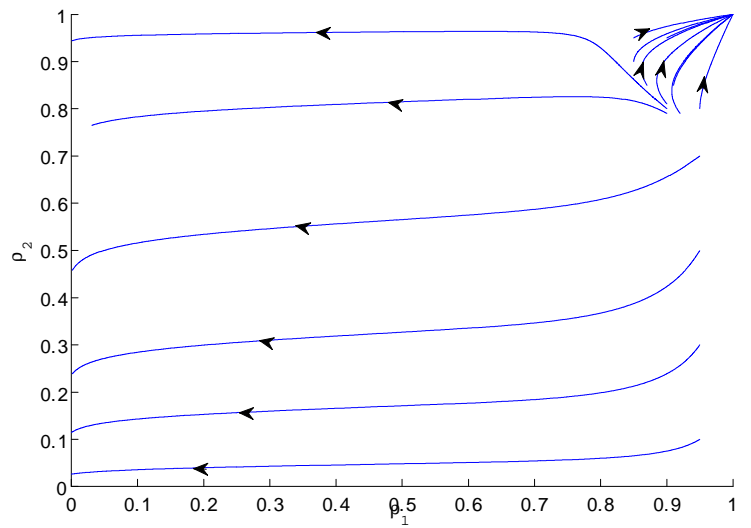

(b) Phase plot $\alpha_{1}=0, \alpha_{2}=9 ; \beta_{1}=0, \beta_{2}=9$

Figure 2: Symmetric non-restrictive and asymmetric thresholds

(c) $\alpha_{1}=\alpha_{2}=\frac{n}{2}-1, \beta_{1}=\beta_{2}=0$. Both type $I$ and type $J$ countries cooperate if all type 
I countries cooperate. This behavioral strategy can be interpreted as an idea that emerged at the Conference of the Parties (COP 15) in Copenhagen in 2009: all countries are willing to cut emissions if all Annex I countries cut emissions. As can be seen in Figure 3a, partially cooperative equilibria of the form $\left(\rho_{1}^{*}, \rho_{2}^{*}\right)$ with $\rho_{1}^{*}<0.2$ occur, even if the two populations start in the all-defection state $\rho_{1}=\rho_{2}=0$.

(d) $\alpha_{1}=\alpha_{2}=\beta_{1}=\beta_{2}=\frac{n}{2}-1$. Both type $I$ and type $J$ countries cooperate if all the other countries cooperate. Note that this case is a generalization of the analysis in Boyd and Richerson (1988) to asymmetric populations. As can be seen in Figure 3b, the long-run attractors are the equilibria $(0,0)$ and $(1,1)$, with a separatrix at a certain fraction of type $J$ countries. Full cooperation is thus possible provided that the reciprocators are very restrictive and that the type $J$ population starts with a sufficiently high fraction of reciprocators.

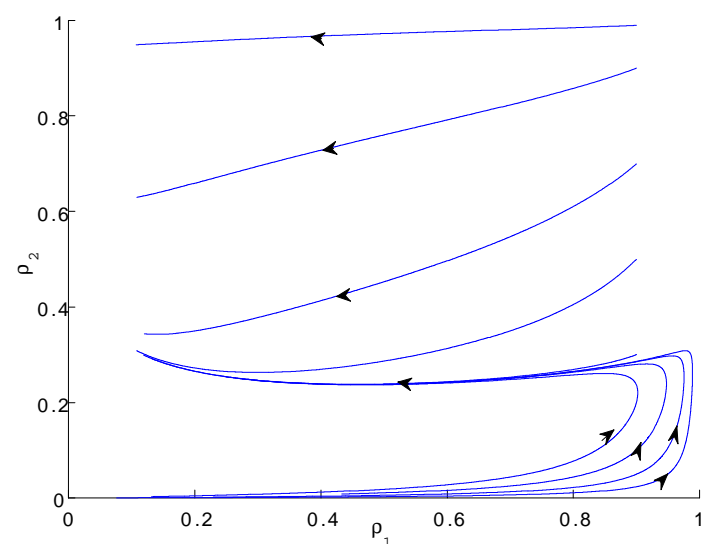

(a) Phase plot $\alpha_{1}=9, \alpha_{2}=9 ; \beta_{1}=0, \beta_{2}=0$

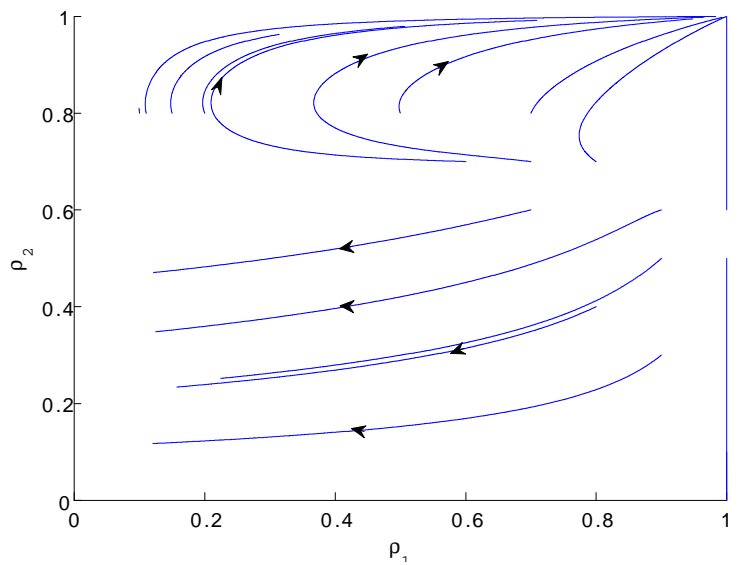

(b) Phase plot $\alpha_{1}=9, \alpha_{2}=9 ; \beta_{1}=9, \beta_{2}=9$

Figure 3: Symmetric partly restrictive and restrictive thresholds

The reciprocal strategy $T F F_{\frac{n}{2}-1, \frac{n}{2}-1}^{I, J}$ seems to be the most promising avenue for inducing cooperation within the asymmetric $n$-player repeated Prisoner's Dilemma game. In the sequel, we will investigate its robustness with respect to some key benchmark parameters. 


\subsection{Sensitivity to benchmark parameters}

First we look at the number of countries $n$ and the probability of an additional round in the repeated game $w$. As can be seen in Figure 4a, increasing the number of countries $n$ worsens the social dilemma, in case of very restrictive thresholds $\left(\alpha_{1}=\alpha_{2}=9 ; \beta_{1}=\beta_{2}=9\right)$. Whereas for a relatively small number of participants $(n=20)$ reciprocity evolves in the two populations, for larger $n$ at some point unconditional defectors take over. As can be seen in Figure 4b, decreasing the probability of an additional round in the repeated game $w$ has a similar effect. For $w$ larger than 0.8, reciprocity evolves in the two populations but for a smaller $w$ the system moves away towards partially cooperative states. It follows that the group should not be too large and should interact sufficiently many times in order to increase reciprocity in the two populations.

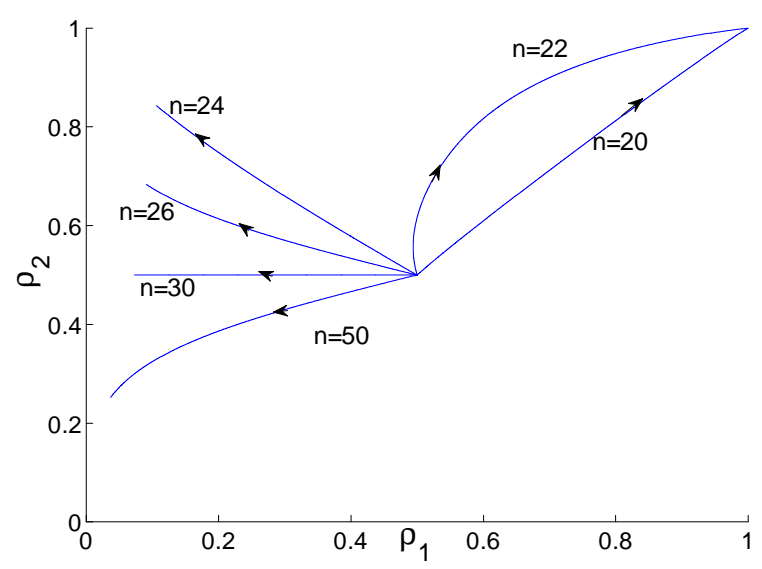

(a) Long-run attractors for different number of countries

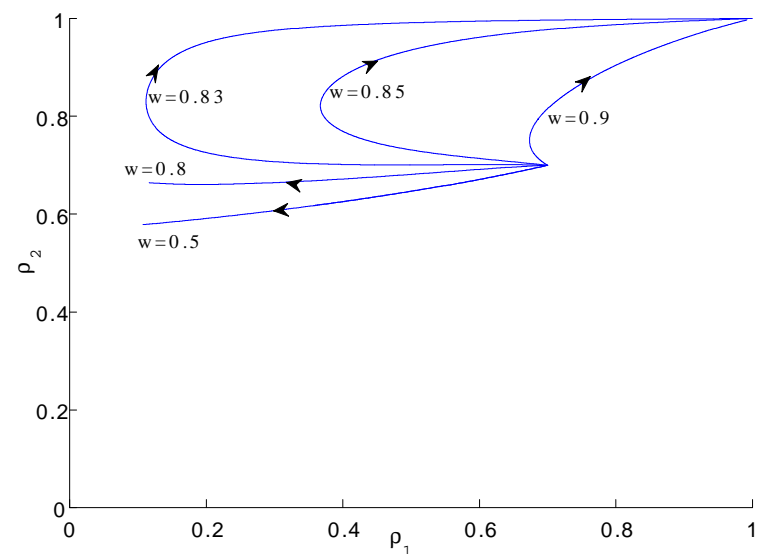

(b) Long-run attractors for different probability of an additional round

Figure 4: Sensitivity to number of countries and probability of an additional round

Next, we investigate the sensitivity with respect to the thresholds $\left(\alpha_{1}, \beta_{1}\right)^{1}$ in the reciprocal strategy $T F T_{\alpha_{1}, \beta_{1}}^{i}$. We start with the initial fractions $\left(\rho_{1}, \rho_{2}\right)$ of reciprocators $(0.75,0.75)$,

\footnotetext{
${ }^{1}$ A similar sensitivity test can be performed with respect to type $J$ triggers $\left(\alpha_{2}, \beta_{2}\right)$ but with fewer degrees of freedom, given the constraint on thresholds $\alpha_{1} \leq \alpha_{2}, \beta_{1} \leq \beta_{2}$ which effectively restricts $\alpha_{2} \in\left[\alpha_{1}, \frac{n}{2}-1\right]$ and $\beta_{2} \in\left[\beta_{1}, \frac{n}{2}-1\right]$.
} 
increase $\alpha_{1} \in\{5,7,8,9\}$ and $\beta_{1} \in\{6,7,8,9\}$, and keep the remaining parameters at $n=$ 20, $\alpha_{2}=9, \beta_{2}=9, B=40, b=30, c_{h}=3, c_{l}=2$ and $w=0.8$. As can be seen in Figure $5 \mathrm{ab}$, unless the reciprocal strategy $T F T_{\alpha_{1}, \beta_{1}}^{i}$ conditions on all other countries cooperating (i.e. $\alpha_{1}=9$ and $\beta_{1}=9$ ) the evolutionary dynamics with asymmetric countries cannot sustain cooperation. However, we have seen that a high probability of continued interaction $w$ improves cooperation. Indeed, as can be seen in Figure $5 \mathrm{~cd}$, for a high probability $(w=0.95)$ the emergence of reciprocators in both populations occurs for lower thresholds $\left(\alpha_{1}<\frac{n}{2}-1\right.$ or $\left.\beta_{1}<\frac{n}{2}-1\right)$. It follows that a combination of sufficiently high initial fractions $\left(\rho_{1}, \rho_{2}\right)$ of reciprocators, sufficiently high thresholds $\left(\alpha_{1}, \beta_{1}\right)$ in the reciprocal strategy $T F T_{\alpha_{1}, \beta_{1}}^{i}$ and a sufficiently high probability of continued interaction $w$ induces full cooperation at the end of the process. 


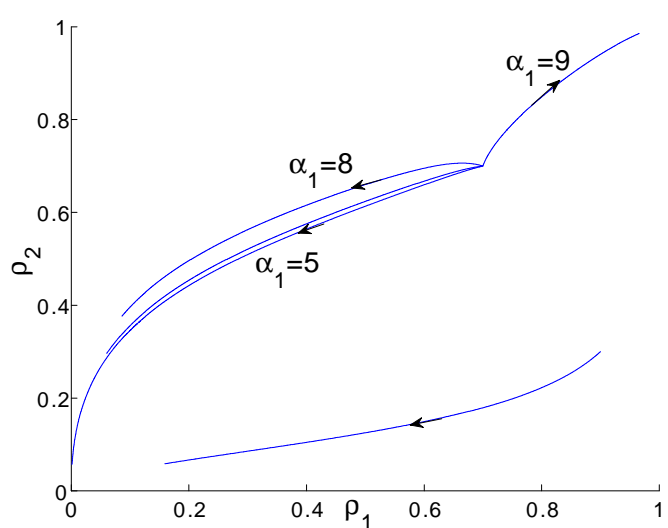

(a) Varying $\alpha_{1}[w=0.8]$

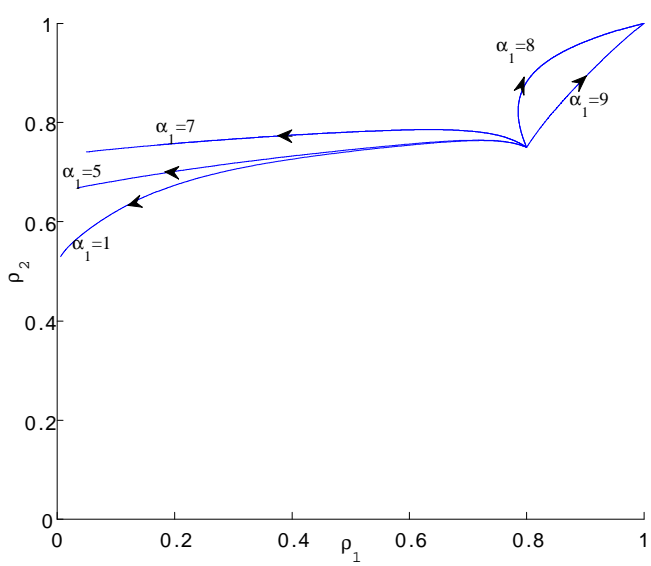

(c) Varying $\alpha_{1}[w=0.95]$

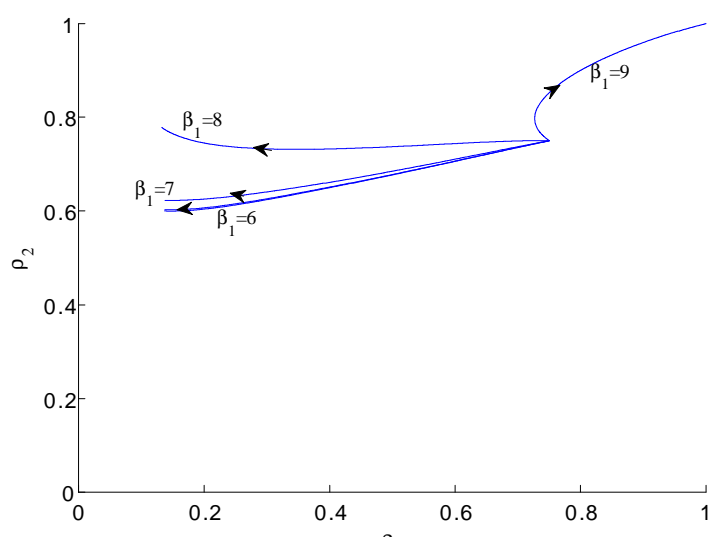

(b) Varying $\beta_{1}[w=0.8]$

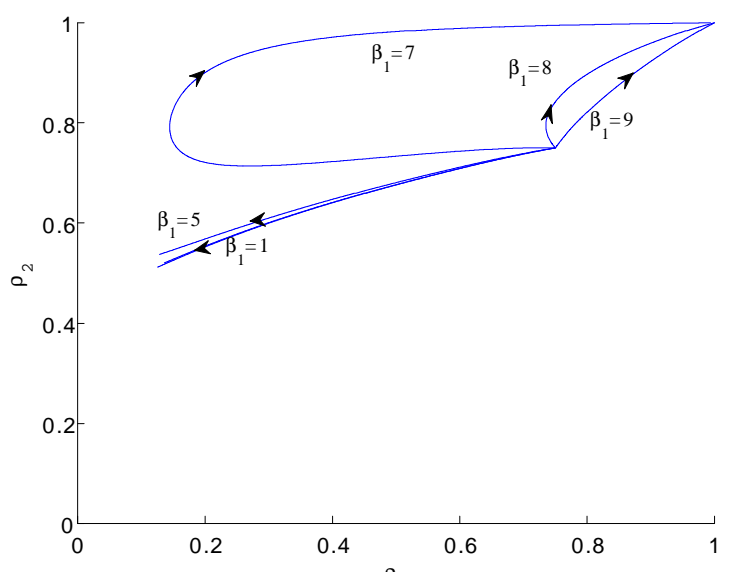

(d) Varying $\beta_{1}[w=0.95]$

Figure 5: Combination of lower thresholds and higher probability of an additional round

Finally, we look at the benefit and cost parameters $B, b, c_{h}$ and $c_{l}$. Changes in these parameters are more involved because the type of asymmetry changes when the order in the benefit and cost parameters changes. So far the numerical analysis has focused on the "typical" $B>b$ and $c_{h}>c_{l}$ asymmetry (labelled scenario I in Figure 6a with parameters $n=20, B=40, b=30, c_{h}=3, c_{l}=2$ and $\left.w=0.8\right)$. One can also imagine, however, that the type of country that may contribute the most to emissions reductions has lower costs (e.g. China in the climate game) so that $B>b$ and $c_{h}<c_{l}$ (labelled scenario II with 
payoff parameters $B=40, b=30, c_{h}=2, c_{l}=3$ ). Figure 6 a depicts the time evolution of the trajectory originating at the initial fractions $\left(\rho_{1}, \rho_{2}\right)=(0.7,0.7)$ of $T F T$ countries for these two cases and for the other two cases where type $I$ countries have the smaller possible contribution and either incur the highest $\operatorname{cost}\left(B=30, b=40, c_{h}=2, c_{l}=3\right.$, labelled scenario III) or the lowest costs $\left(B=30, b=40, c_{h}=2, c_{l}=3\right.$, labelled scenario IV $)$. Our theoretical framework and its ensuing numerical analysis allow for the investigation of these reversals in the asymmetry. Cases I and III appear to be the most conducive to full cooperation (convergence to equilibrium $(1,1)$ ). Case II may generate a partially cooperative state of the form $\left(\rho_{1}^{*}, 0\right), \rho_{1}^{*}>0$, whereas case IV appears as the most detrimental co-evolution of reciprocators, with both populations converging to the AllD state. Note, however, that even in this worst case scenario a positive outcome may still be reached by pushing up the probability of continued interaction $w$, as can be seen in Figure $6 \mathrm{~b}$.

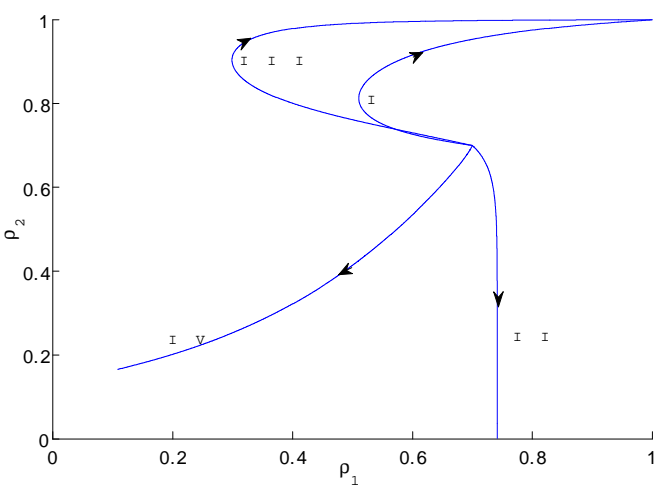

(a) Long-run attractors for different payoff asymmetries

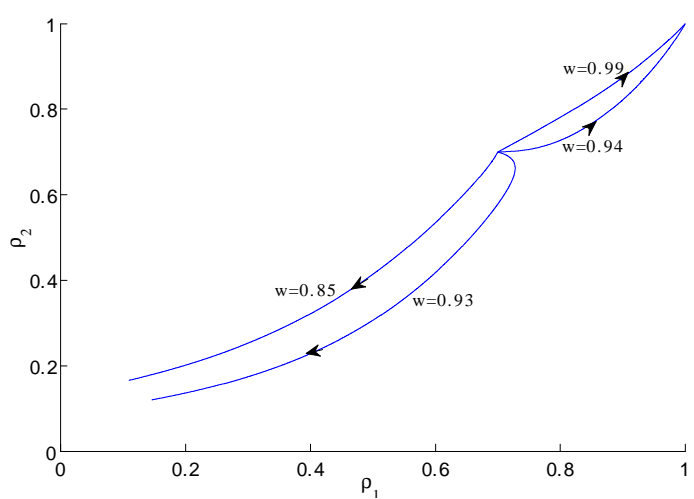

(b) Scenario IV for different probability of an additional round

Figure 6: Sensitivity to payoff asymmetries

\section{Conclusions}

Reciprocity is a key mechanism in inducing cooperation in asymmetric two-player repeated

Prisoner's Dilemma games and may therefore provide a possible way-out of the problems in 
international environmental negotiations such as the Kyoto Protocol on climate change. In this paper we use a typical model for International Environmental Agreements but analyze a different institutional setting where countries may use reciprocal trigger strategies instead of trying to reach a unanimous agreement. Since a large number of countries is involved and countries are not identical, we had to extend the basic theory to more than two players and to asymmetries in benefits and costs and in the thresholds of the trigger strategies.

Basically we investigated whether in a population of countries that consists of reciprocators and unconditional defectors the fraction of reciprocators can grow because they are successful, so that cooperation will increase. We focused on the case with two types of countries: the first type has high emissions and high costs of emission reductions and the other type has low emissions and low costs of emission reductions. Groups of countries are randomly drawn from the population and interact in a $n$-player repeated Prisoner's Dilemma game. Successful strategies are imitated in replicator dynamics and in this way the fraction of reciprocators may increase but may also decrease. Our results show that there exist regions in the parameter space such that (partial) cooperation may emerge as the long-run attractor of an evolutionary selection process. We show that full cooperative behavior may be sustained if there exists a critical mass of initial cooperators, if the thresholds for the trigger strategies are high and if the duration of the repeated interaction is sufficiently long.

In the context of the Conference of the Parties on climate change, it may help if countries switch to "conditioning" behavior when pledging a certain fraction of emission reductions, instead of trying to reach a unanimous agreement. An example is the European Union strategy of stepping up to a $30 \%$ emission reduction provided that other developed countries match this contribution and developing countries take "appropriate" actions. Numerical simulations of our model suggest that, in the highly asymmetric context of the climate game, this strategy may be successful provided that developed and developing countries are willing to make similar pledges and provided that they are interacting repeatedly and initiating this process with a sufficiently large group of countries. 


\section{References}

Barrett, S. (1994). Self-enforcing international environmental agreements. Oxford Economic Papers 46, 878-894.

Boyd, R. and P. Richerson (1988). The evolution of reciprocity in sizable groups. Journal of Theoretical Biology 132, 337-356.

Breton, M., L. Sbragia, and G. Zaccour (2010). A dynamic model for international environmental agreements. Environmental and Resource Economics 45(1), 25-48.

Diekmann, A. and K. Manhart (1989). Cooperation in 2 and n-person prisoner's dilemma games: a simulation study. Analyse and Kritik 11, 134-153.

Finus, M. (2003). Stability and design of international environmental agreements: the case of transboundary pollution. In H. Folmer and T. Tietenberg (Eds.), The International Yearbook of Environmental and Resource Economics 2003/2004: a Survey of Current Issues, pp. 82-158. Cheltenham: Edward Elgar.

Fuentes-Albero, C. and S. Rubio (2010). Can international environmental cooperation be bought? European Journal of Operations Research 202(1), 255-264.

Hoel, M. and A. de Zeeuw (2013). Technology agreements with heterogeneous countries. CREE Working paper 1/2013.

Lindgren, K. and L. Johanson (2002). Coevolution of strategies in n-person prisoner's dilemma. In J. P. Crutchfield and P. Schuster (Eds.), Evolutionary Dynamics: Exploring the Interplay of Selection, Accident, Neutrality, and Function, Santa Fe Institute Studies in the Sciences of Complexity. Santa Fe Institute.

McGinty, M. (2007). International environmental agreements among asymmetric nations. Oxford Economic Papers 59(1), 45-62. 
McGinty, M. (2010). International environmental agreements as evolutionary games. Environmental and Resource Economics 45, 251-269.

Molander, P. (1992). The prevalence of free-riding. Journal of Conflict Resolution 36(4), $756-771$.

Nowak, M. and K. Sigmund (2006). Five rules for the evolution of cooperation. Science 314, $1560-1563$.

Pavlova, Y. and A. de Zeeuw (2013). Asymmetries in international environmental agreements. Environment and Development Economics 18(1), 51-68.

Rieskamp, J. and P. Todd (2006). The evolution of cooperative strategies for asymmetric social interactions. Theory and Decision 60, 69-111.

Schelling, T. (1978). Micromotives and Macrobehavior. W. W. Norton and Co.

Taylor, M. (1976). Anarchy and cooperation. John Wiley \& Sons. 


\section{Appendix A Repeated game payoff differentials}

The replicator dynamics (6)-(7) is driven by the payoff differentials $\Delta \Pi_{i}=\Pi_{T F T_{\alpha_{1}, \beta_{1}}^{i}}-\Pi_{A l l D^{i}}$. We focus in the sequel on case (i) in Section 2.2, i.e. when the conditioning behavior of a type $J$ country is more demanding with respect to cooperation of both types of countries: $\left\{\alpha_{1} \leq \alpha_{2}, \beta_{1} \leq \beta_{2}\right\}$. Fig. (1) displays the possible paths of actions of the countries using TFT strategies.

Given the fractions $\left(\rho_{1}, \rho_{2}\right)$ of reciprocators in the populations $I, J$, the number of trigger strategies TFT in a sample of size $\frac{n}{2}$ is binomially distributed:

$$
\begin{aligned}
& \text { - \# of } T F T_{\alpha_{1}, \beta_{1}}^{i} \text { in a sample of } \frac{n}{2} \equiv k \sim B_{1}\left(\frac{n}{2} \rho_{1}, \frac{n}{2} \rho_{1}\left(1-\rho_{1}\right)\right) \text {, } \\
& \text { - \# of } T F T_{\alpha_{2}, \beta_{2}}^{j} \text { in a sample of } \frac{n}{2} \equiv l \sim B_{2}\left(\frac{n}{2} \rho_{2}, \frac{n}{2} \rho_{2}\left(1-\rho_{2}\right)\right) \text {. }
\end{aligned}
$$

Figure (1) shows that the space $(k, l)$ can be split in three payoff-equivalent regions that we denote by $R_{1}, R_{2}, R_{3}$. We first compute $\Pi_{T F T_{\alpha_{1}, \beta_{1}}^{i}}, \Pi_{A l l D^{i}}$ and $\Delta \Pi_{i}$ for each region.

\section{A.1 $\quad R_{1} \equiv\left\{(k, l), k \geq \alpha_{2}, l \geq \beta_{2}\right\}$}

In region 1 , both types $I, J$ start with and continue cooperation $C$. For type $I$, the expected net benefit of the TFT strategy is

$$
\begin{aligned}
\Pi_{T F T_{\alpha_{1}, \beta_{1}}^{i}}^{1} & =\sum_{k=\alpha_{2}}^{\frac{n}{2}-1} B_{1}(k) \sum_{l=\beta_{2}}^{\frac{n}{2}} B_{2}(l) \Pi_{i}^{1}\left(C_{i}|k+1| l\right) \\
& =\sum_{k=\alpha_{2}}^{\frac{n}{2}-1} B_{1}(k) \sum_{l=\beta_{2}}^{\frac{n}{2}} B_{2}(l) \frac{V\left(C_{i}|k+1| l\right)}{1-w} \\
& =\sum_{k=\alpha_{2}}^{\frac{n}{2}-1} B_{1}(k) \sum_{l=\beta_{2}}^{\frac{n}{2}} B_{2}(l) \frac{1}{1-w}\left(\frac{B(k+1)+b l}{n}-c_{h}\right),
\end{aligned}
$$


whereas the AllD strategy yields

$$
\Pi_{A l l D^{i}}^{1}=\sum_{k=\alpha_{2}}^{\frac{n}{2}-1} B_{1}(k) \sum_{l=\beta_{2}}^{\frac{n}{2}} B_{2}(l) \Pi_{i}^{1}\left(D_{i}|k| l\right)
$$

with

$$
\Pi_{i}^{1}\left(D_{i}|k| l\right)=\left\{\begin{array}{c}
V\left(D_{i}|k| l\right)+\frac{w V\left(D_{i}|k| 0\right)}{1-w}=\frac{B k+b l}{n}+\frac{w}{1-w} \frac{B k}{n}, \text { if }\left(k=\alpha_{2}\right) \wedge\left(l=\beta_{2}\right) \\
\frac{V\left(D_{i}|k| l\right)}{1-w}=\frac{1}{1-w} \frac{B k+b l}{n}=\frac{B k+b l}{n}+\frac{w}{1-w} \frac{B k+b l}{n}, \text { otherwise. }
\end{array}\right\} .
$$

Combining $\Pi_{T F T_{\alpha_{1}, \beta_{1}}^{i}}^{1}$ and $\Pi_{A l l D^{i}}^{1}$ we can preserve both lower bounds of the summation operators as long as we adjust for the bordercase ${ }^{2}$ payoffs at $\left(k=\alpha_{2}\right)$ and $\left(l=\beta_{2}\right)$ :

$$
\begin{gathered}
\Pi_{T F T_{\alpha_{1}, \beta_{1}}^{i}}^{1}-\Pi_{A l l D^{i}}^{1}=\sum_{k=\alpha_{2}}^{\frac{n}{2}-1} B_{1}(k) \sum_{l=\beta_{2}}^{\frac{n}{2}-1} B_{2}(l)\left[\frac{V\left(C_{i}|k+1| l\right)}{1-w}-\frac{V\left(D_{i}|k| l\right)}{1-w}\right]+ \\
+B_{1}\left(\alpha_{2}\right) B_{2}\left(\beta_{2}\right)\left\{\left[\frac{V\left(D_{i}|k| l\right)}{1-w}\right]-\left[V\left(D_{i}|k| l\right)+\frac{w}{1-w} V\left(D_{i}|k| 0\right)\right]\right\}_{k=\alpha_{2}, l=\beta_{2}}
\end{gathered}
$$

Finally, by using the expressions for $V\left(C_{i}|k+1| l\right), V\left(D_{i}|k| l\right)$ and $V\left(D_{i}|k| 0\right)$, we obtain region 1's payoff difference:

$$
\begin{aligned}
\Delta \Pi_{i}^{1}= & \frac{1}{1-w}\left\{\left(\frac{B}{n}-c_{h}\right)\left[1-I_{\rho_{1}}\left(\alpha_{2}+1, \frac{n}{2}-1-\alpha_{2}\right)\right]\left[1-I_{\rho_{2}}\left(\beta_{2}+1, \frac{n}{2}-\beta_{2}\right)\right]\right\}+ \\
& +B_{1}\left(\alpha_{2}\right) B_{2}\left(\beta_{2}\right)\left(\frac{w b \beta_{2}}{n}\right),
\end{aligned}
$$

where $I_{\rho_{1}}(\cdot, \cdot), I_{\rho_{2}}(\cdot, \cdot)$ stand for the regularized incomplete beta functions ${ }^{3}$.

\footnotetext{
${ }^{2}$ We assume that type $j$ player continues to cooperate unless both $k=\alpha_{2}$ and $l=\beta_{2}$ hold. This assumption is not innocuous as a more stringent strategy (i.e. start cooperate and revert to perpetual defection if either $k=\alpha_{2}$ or $l=\beta_{2}$ ) would lead to a different payoff structure for the AllD strategy. A similar assumption applies when the second corner point is hit: $k=\alpha_{1}$ and $l=\beta_{1}$.

${ }^{3}$ The incomplete beta function is given by: $B(x ; a, b)=\int_{0}^{x} t^{a-1}(1-t)^{b-1} d t$ which can be normalized by the (complete) beta function $B(a, b)=\int_{0}^{1} t^{a-1}(1-t)^{b-1} d t$ to obtain the regularized imcomplete beta function $I_{x}(a, b)=\frac{B(x ; a, b)}{B(a, b)}$. For a binomially distributed random variable $X \sim B(p, n)$ the regularized beta function $I$ characterizes the cumulative probability distribution, i.e. $P(X \leq \alpha)=I_{1-p}(n-\alpha, \alpha+1)=1-I_{p}(\alpha+1, n-\alpha)$.
} 


\section{A.2 $\quad R_{2} \equiv\left\{(k, l),\left(k \geq \alpha_{1} \wedge l \geq \beta_{1}\right) \backslash R_{1}\right\}$}

In region 2, type $I$ starts with and continues cooperation $C$, while type $J$ starts with cooperation $C$ but switches to defection $D$. The expected net benefits of a $T F T$ strategy from population $I$ are

$$
\Pi_{T F T_{\alpha_{1}, \beta_{1}}^{i}}^{2}=\sum_{k=\alpha_{1}}^{\alpha_{2}-1} B_{1}(k) \sum_{l=\beta_{1}}^{\frac{n}{2}} B_{2}(l) \Pi_{i}^{2}\left(C_{i}|k+1| l\right)+\sum_{k=\alpha_{2}}^{\frac{n}{2}-1} B_{1}(k) \sum_{l=\beta_{1}}^{\beta_{2}} B_{2}(l) \Pi_{i}^{2}\left(C_{i}|k+1| l\right)
$$

with

$$
\begin{gathered}
\Pi_{i}^{2}\left(C_{i} \quad|\quad k+1| l\right)=V\left(C_{i}|k+1| l\right)+\frac{w}{1-w} V\left(C_{i}|k+1| 0\right) \\
=\left(\frac{B(k+1)+b l}{n}-c_{h}\right)+\frac{w}{1-w}\left(\frac{B(k+1)}{n}-c_{h}\right),
\end{gathered}
$$

whereas the expected net benefits accruing to the AllD strategy are given by

$$
\Pi_{A l l D^{i}}^{2}=\sum_{k=\alpha_{1}}^{\alpha_{2}-1} B_{1}(k) \sum_{l=\beta_{1}}^{\frac{n}{2}} B_{2}(l) \Pi_{i}^{2}\left(D_{i}|k| l\right)+\sum_{k=\alpha_{2}}^{\frac{n}{2}-1} B_{1}(k) \sum_{l=\beta_{1}}^{\beta_{2}} B_{2}(l) \Pi_{i}^{2}\left(D_{i}|k| l\right)
$$

with

$$
\Pi_{i}^{2}\left(D_{i}|k| l\right)=\left\{\begin{array}{c}
V\left(D_{i}|k| l\right)+\frac{w V\left(D_{i}|0| 0\right)}{1-w}=\frac{B k+b l}{n}+\frac{w}{1-w} 0, \text { if }\left(k=\alpha_{1}\right) \wedge\left(l=\beta_{1}\right) \\
V\left(D_{i}|k| l\right)+\frac{w V\left(D_{i}|k| 0\right)}{1-w}=\frac{B k+b l}{n}+\frac{w}{1-w} \frac{B k}{n} \text {, otherwise. }
\end{array}\right\} .
$$

Combining $\Pi_{T F T_{\alpha_{1}, \beta_{1}}^{i}}^{2}$ and $\Pi_{A l l D^{i}}^{2}$, and accounting for the bordercase $\left(k=\alpha_{1}\right) \wedge\left(l=\beta_{1}\right)$, 
we obtain for $\Delta \Pi_{i}^{2}=\Pi_{T F T_{\alpha_{1}, \beta_{1}}^{i}}^{2}-\Pi_{A l l D^{i}}^{2}$ :

$$
\begin{aligned}
& \Delta \Pi_{i}^{2}=\sum_{k=\alpha_{1}}^{\alpha_{2}-1} B_{1}(k) \sum_{l=\beta_{1}}^{\frac{n}{2}} B_{2}(l)\left\{\left[V\left(C_{i}|k+1| l\right)+\frac{w}{1-w} V\left(C_{i}|k+1| 0\right)\right]-V\left(D_{i}|k| l\right)-\frac{w V\left(D_{i}|k| 0\right)}{1-w}\right\} \\
& +\sum_{k=\alpha_{2}}^{\frac{n}{2}-1} B_{1}(k) \sum_{l=\beta_{1}}^{\beta_{2}} B_{2}(l)\left\{\left[V\left(C_{i}|k+1| l\right)+\frac{w}{1-w} V\left(C_{i}|k+1| 0\right)\right]-V\left(D_{i}|k| l\right)-\frac{w V\left(D_{i}|k| 0\right)}{1-w}\right\} \\
& -B_{1}\left(\alpha_{1}\right) B_{2}\left(\beta_{1}\right)\left\{\left[V\left(D_{i}|k| l\right)+\frac{w V\left(D_{i}|k| 0\right)}{1-w}\right]-\left[V\left(D_{i}|k| l\right)+\frac{w V\left(D_{i}|0| 0\right)}{1-w}\right]\right\}_{k=\alpha_{1}, l=\beta_{1} .}
\end{aligned}
$$

Using (1), (2) for $V\left(C_{i}|k+1| l\right), V\left(C_{i}|k+1| 0\right), V\left(D_{i}|k| l\right)$ and $V\left(D_{i}|0| 0\right)$ we can simplify, with the help of the incomplete beta function to

$$
\begin{aligned}
\Delta \Pi_{i}^{2}= & \frac{1}{1-w}\left\{( \frac { B } { n } - c _ { h } ) [ 1 - I _ { \rho _ { 2 } } ( \beta _ { 1 } + 1 , \frac { n } { 2 } - \beta _ { 1 } ) ] \left[I_{\rho_{1}}\left(\alpha_{2}+1, \frac{n}{2}-1-\alpha_{2}\right)\right.\right. \\
& \left.\left.-I_{\rho_{1}}\left(\alpha_{1}+1, \frac{n}{2}-1-\alpha_{1}\right)\right]\right\}+B_{1}\left(\alpha_{1}\right) B_{2}\left(\beta_{1}\right)\left(\frac{w B \alpha_{1}}{n}-\frac{b \beta_{1}}{n}\right) .
\end{aligned}
$$

\section{A.3 $R_{3} \equiv\left\{(k, l),(k \geq 0 \wedge l \geq 0) \backslash\left\{R_{1} \cup R_{2}\right\}\right\}$}

In region 3 , both types $I, J$ start with cooperation $C$ and switch to defection $D$. The expected net benefits of the TFT strategy from population $I$ are

$$
\Pi_{T F T_{\alpha_{1}, \beta_{1}}^{i}}^{3}=\sum_{k=0}^{\alpha_{1}-1} B_{1}(k) \sum_{l=0}^{\frac{n}{2}} B_{2}(l) \Pi_{i}^{3}\left(C_{i}|k+1| l\right)+\sum_{k=\alpha_{1}}^{\frac{n}{2}-1} B_{1}(k) \sum_{l=0}^{\beta_{1}} B_{2}(l) \Pi_{i}^{3}\left(C_{i}|k+1| l\right)
$$

with

$$
\Pi_{i}^{3}\left(C_{i}|k+1| l\right)=V\left(C_{i}|k+1| l\right)+\frac{w}{1-w} V\left(C_{i}|0| 0\right)=\frac{B(k+1)+b l}{n}-c_{h}
$$

whereas $A l l D$ strategies from population $I$ get

$$
\Pi_{A l l D^{i}}^{3}=\sum_{k=0}^{\alpha_{1}-1} B_{1}(k) \sum_{l=0}^{\frac{n}{2}} B_{2}(l) \Pi_{i}^{3}\left(D_{i}|k| l\right)+\sum_{k=\alpha_{1}}^{\frac{n}{2}-1} B_{1}(k) \sum_{l=0}^{\beta_{1}} B_{2}(l) \Pi_{i}^{3}\left(D_{i}|k| l\right)
$$


with

$$
\Pi_{i}^{3}\left(D_{i}|k| l\right)=V\left(D_{i}|k| l\right)+\frac{w V\left(D_{i}|0| 0\right)}{1-w}=\frac{B k+b l}{n} .
$$

Combining $\Pi_{T F T_{\alpha_{1}, \beta_{1}}^{i}}^{3}$ and $\Pi_{A l l D^{i}}^{3}$, we can write the repeated game's payoff difference in region 3 as

$$
\Delta \Pi_{i}^{3}=\left(\frac{B}{n}-c_{h}\right)\left[I_{\rho_{1}}\left(\alpha_{2}+1, \frac{n}{2}-1-\alpha_{2}\right)+I_{\rho_{2}}\left(\beta_{1}+1, \frac{n}{2}-\beta_{1}\right)\right]\left[1-I_{\rho_{1}}\left(\alpha_{1}+1, \frac{n}{2}-1-\alpha_{1}\right)\right] .
$$

\section{A.4 Payoff differentials}

Summing up the results for the three regions (9), (10) and (11), the expected payoff difference between a type $I$ country using strategy $T F T_{\alpha_{1}, \beta_{1}}^{i}$ and an unconditional defector $A l l D^{i}$ yields

$$
\begin{aligned}
\Delta \Pi_{i}= & \frac{1}{1-w}\left\{\left(\frac{B}{n}-c_{h}\right)\left[1-I_{\rho_{1}}\left(\alpha_{2}+1, \frac{n}{2}-1-\alpha_{2}\right)\right]\left[1-I_{\rho_{2}}\left(\beta_{2}+1, \frac{n}{2}-\beta_{2}\right)\right]+\right. \\
& +\left[1-I_{\rho_{2}}\left(\beta_{1}+1, \frac{n}{2}-\beta_{1}\right)\right]\left[I_{\rho_{1}}\left(\alpha_{2}+1, \frac{n}{2}-1-\alpha_{2}\right)-I_{\rho_{1}}\left(\alpha_{1}+1, \frac{n}{2}-1-\alpha_{1}\right)\right] \\
& +(1-w)\left[I_{\rho_{1}}\left(\alpha_{2}+1, \frac{n}{2}-1-\alpha_{2}\right)+I_{\rho_{2}}\left(\beta_{1}+1, \frac{n}{2}-\beta_{1}\right)\right]\left[1-I_{\rho_{1}}\left(\alpha_{1}+1, \frac{n}{2}-1-\alpha_{1}\right)\right] \\
& \left.+(1-w) B_{1}\left(\alpha_{1}\right) B_{2}\left(\beta_{1}\right)\left(\frac{w B \alpha_{1}}{n}-\frac{b \beta_{1}}{n}\right)+(1-w) B_{1}\left(\alpha_{2}\right) B_{2}\left(\beta_{2}\right)\left(\frac{w b \beta_{2}}{n}\right)\right\} .
\end{aligned}
$$

A similar computation yields type $J$ 's payoff differential between $T F T_{\alpha_{2}, \beta_{2}}^{j}$ and $A l l D^{j}$ :

$$
\begin{aligned}
\Delta \Pi_{j}= & \frac{1}{1-w}\left\{\left(\frac{b}{n}-c_{l}\right)\left[1-I_{\rho_{1}}\left(\alpha_{2}+1, \frac{n}{2}-\alpha_{2}\right)\right]\left[1-I_{\rho_{2}}\left(\beta_{2}+1, \frac{n}{2}-1-\beta_{2}\right)\right]\right. \\
& +(1-w)\left[1-I_{\rho_{2}}\left(\beta_{1}+1, \frac{n}{2}-1-\beta_{1}\right)\right]\left[I_{\rho_{1}}\left(\alpha_{2}+1, \frac{n}{2}-\alpha_{2}\right)-I_{\rho_{1}}\left(\alpha_{1}+1, \frac{n}{2}-\alpha_{1}\right)\right] \\
& +(1-w)\left[I_{\rho_{1}}\left(\alpha_{2}+1, \frac{n}{2}-\alpha_{2}\right)+I_{\rho_{2}}\left(\beta_{1}+1, \frac{n}{2}-1-\beta_{1}\right)\right]\left[1-I_{\rho_{1}}\left(\alpha_{1}+1, \frac{n}{2}-\alpha_{1}\right)\right] \\
& \left.+(1-w) B_{1}\left(\alpha_{1}\right) B_{2}\left(\beta_{1}\right)\left(\frac{w B \alpha_{1}}{n}-\frac{b \beta_{1}}{n}\right)+(1-w) B_{1}\left(\alpha_{2}\right) B_{2}\left(\beta_{2}\right)\left(\frac{w b \beta_{2}}{n}\right)\right\} .
\end{aligned}
$$

